\title{
Comparative analysis of NMR chemical shift predictions for proteins in the solid phase
}

\author{
Karsten Seidel $^{\mathrm{a}}$, Manuel Etzkorn ${ }^{\mathrm{a}}$, Robert Schneider ${ }^{\mathrm{a}}$, Christian Ader ${ }^{\mathrm{a}, \mathrm{b}}$, Marc Baldus ${ }^{\mathrm{a}, \mathrm{b}, *}$ \\ a Max Planck Institute for Biophysical Chemistry, Am Fassberg 11, 37077 Göttingen, Germany \\ ${ }^{\mathrm{b}}$ Bijvoet Centre for Biomolecular Research, Utrecht University, $3584 \mathrm{CH}$ Utrecht, The Netherlands
}

\section{A R T I C L E I N F O}

\section{Article history:}

Received 13 August 2008

Received in revised form

10 December 2008

Available online 20 January 2009

Keywords:

Solid state

Chemical shift

Prediction

SHIFTS

PROSHIFT

SHIFTX

SPARTA

Membrane protein

\begin{abstract}
A B S T R A C T
A comparative analysis of nuclear chemical shift predictions of proteins in the solid state by rapid algorithms trained on and verified with solution-state NMR assignments is presented. The precision of predictions by four dedicated computer programs (SHIFTS, PROSHIFTS, SHIFTX and SPARTA) was found to be close to values obtained for proteins in solution. Correlation coefficients depend on the NMR nucleus $\left(\mathrm{N}, \mathrm{C}^{\prime}, \mathrm{C}^{\alpha}\right.$ and $\mathrm{C}^{\beta}$ ) and on secondary structure ( $\beta$-strand, random coil and $\alpha$-helix), but also on the molecular environment (membrane-integral or not). The findings establish a quantitative basis for using chemical shift prediction programs for solid-state NMR applications. On the other hand, prediction inaccuracies identified for certain resonance kind, residue type, and molecular environment point to possible areas of methodological improvement.
\end{abstract}

(c) 2009 Elsevier Inc. All rights reserved.

\section{Introduction}

Physical properties of a system can be assessed by comparing experimental data to calculations from theory. A particularly sensitive probe to local molecular structure is the nuclear chemical shift measured by NMR spectroscopy. Backbone ${ }^{1} \mathrm{H}$, ${ }^{15} \mathrm{~N}$ and ${ }^{13} \mathrm{C}$ chemical shifts depend on several factors, such as hydrogen bonds, electric field effects, ring currents, and backbone and side-chain torsion angles. The influence of intra-residue backbone torsion angles $\phi$ and $\psi$ dominates the chemical shift of $C^{\prime}, C^{\alpha}$ and $C^{\beta}$ with respect to the residue-specific average (secondary chemical shift) [1-5]. ${ }^{15} \mathrm{~N}$ chemical shifts depend also on the conformation of the preceding residue, correlating with $\psi_{i-1}$ in addition to $\phi_{i}$, where $i$ is the residue number of ${ }^{15} \mathrm{~N}[2,6,7]$. The contributions to ${ }^{1} \mathrm{H}$ chemical shifts shall not be considered here.

$A b$ initio calculations of nuclear chemical shifts for entire proteins are generally precluded due to high computational demands. In recent years, however, a number of algorithms became available which allow for a rapid estimation of chemical shifts from existing structural data, based on a combination of quantum chemical approximations or empirical considerations

\footnotetext{
* Corresponding author at: Bijvoet Centre for Biomolecular Research, Utrecht University, 3584 CH Utrecht, The Netherlands. Fax: +31302537623.

E-mail address: m.baldus@uu.nl (M. Baldus).
}

[8-12]. Despite limited precision, these shift predictions can be of great value in structural investigations by NMR spectroscopy. Under magic angle spinning or fast isotropic motion, for example, knowledge on the isotropic chemical shifts in a protein allows for an initial verification of correct overall fold and domain mobility by means of multidimensional correlation spectroscopy. In structural studies on systems with partially known components, such as membrane proteins with solvent-exposed globular domains, shift predictions can help to identify regions of significant structural difference. More recently, rapid chemical shift prediction algorithms have also been used for spectral fitting within structure calculation frameworks [13-15].

Today's chemical shift prediction algorithms have been trained with solution-state NMR assignments, and X-ray crystallographic or solution-state NMR structural data. The quality of predictions was evaluated in reference to soluble proteins. In principle, such predictions should also be valid for proteins in the solid state, when line widths are small compared to the standard error of predictions, and only the isotropic average of the chemical shielding tensor is of interest. On the other hand, sample packing effects or, in a more general sense, the molecular environment can influence the chemical shift in various ways, such as by hydrogen bonding, ring currents, electrostatic interactions, or structural rearrangements. Due to the limited availability of solid-state NMR resonance assignments, the validity of applying these routines in chemical shift predictions for proteins in the solid phase could so far only be evaluated for three micro-crystalline proteins [13]. 
With an increasing number of solid-state NMR resonance assignments, a more systematic investigation becomes possible. For example, Fig. 1A shows a comparison of predictions from an ensemble of structures for transmembrane helix residues N30-I48 of AFA-mutant Phospholamban (AFA-PLN), reconstituted in liposomes [16], to an experimental ${ }^{13} \mathrm{C}$ double-quantum/ single-quantum (2Q/1Q) correlation spectrum. While predictions for Ala, Asn, Leu and Phe residues center at the correlations of the isotropic chemical shifts, Ile predictions deviate by more than one resonance line width from the unambiguously identified cross peak of Ile residues. This observation raises the following questions: How reliable are solid-state NMR chemical-shift predictions using software trained on solution-state NMR data? What are the error margins, and can predictions be improved by correcting for possible effects from the molecular environment? Insights into the dependence between measured and predicted chemical shift may also be of relevance for the inverse process, i.e., the prediction of backbone-torsion angles from chemical shifts by programs such as TALOS [17] or PREDITOR [18]. Possibly, the analysis should not only be based on a larger number of assignments than in Ref. [13], but also on a greater variety of sample preparations.

Here, the performance of four computer programs, SHIFTS (2002) [9], PROSHIFT (2003) [10], SHIFTX (2003) [11] and SPARTA (2007) [12], that allow for the prediction of backbone ${ }^{15} \mathrm{~N}$ and ${ }^{13} \mathrm{C}^{\prime}$, ${ }^{13} \mathrm{C}^{\alpha}$, and side chain ${ }^{13} \mathrm{C}^{\beta}$ (isotropic) NMR chemical shifts at once, was evaluated. For this purpose, a database of more than 600 protein residues was compiled, for which solid-state NMR resonance assignments and high-resolution 3D structures are available. In particular, several proteins with membrane-integral $\alpha$-helices $(m \alpha)$ were included, see Fig. 1B. All four considered programs require three-dimensional input coordinates for the polypeptide of interest. The quality of ${ }^{1} \mathrm{H}$ predictions $\left({ }^{1} \mathrm{H}_{\mathrm{N}},{ }^{1} \mathrm{H}^{\alpha}\right.$ for all four programs, and side-chain ${ }^{1} \mathrm{H}$ for SHIFTS and SHIFTX) has not been considered, as the number of ssNMR assignments for these nuclei is currently very small.

\section{Methods}

Solid-state NMR assignments and 3D structures were retrieved from the BioMagResBank (BMRB) [19], the Protein Data Bank (PDB) [20], from published articles, or directly from the authors, see Table 1 . Chemical shift predictions were obtained from the SHIFTS 4.1.1, PROSHIFT and SHIFTX web servers, and with the SPARTA 2007.05.09 Linux executable. Secondary structure specific chemical shifts were obtained from Ref. [21].

In case of Phospholamban and SRII, where assignments from mobility-selective scalar- and dipolar-based transfer schemes were available, only data for the rigid segments was used. Only shifts that can be predicted by all four programs were considered. Hence, ${ }^{15} \mathrm{~N}$ of Pro residues, which is not predicted by SHIFTX, and all Cys resonances, which are not predicted by SHIFTS, were excluded. Also, amino- and carboxy-terminal residues were discarded, as they exhibit unusual shifts, and are not predicted by SHIFTS and SPARTA. Twelve predictions of $\mathrm{C}^{\prime}$ resonances by SHIFTS, of which eight were assigned in the solid state, were off by about a factor of two, and were excluded from the database [22]. One further unusual shift, ${ }^{15} \mathrm{~N}$ in $\mathrm{Crh}$ Q3 $(82.6 \mathrm{ppm})$, which was $36 \mathrm{ppm}$ off the average prediction of all four programs, was also excluded. In proteins with resonance splitting (Crh, Ubiquitin), only the first resonance (generally corresponding to the stronger
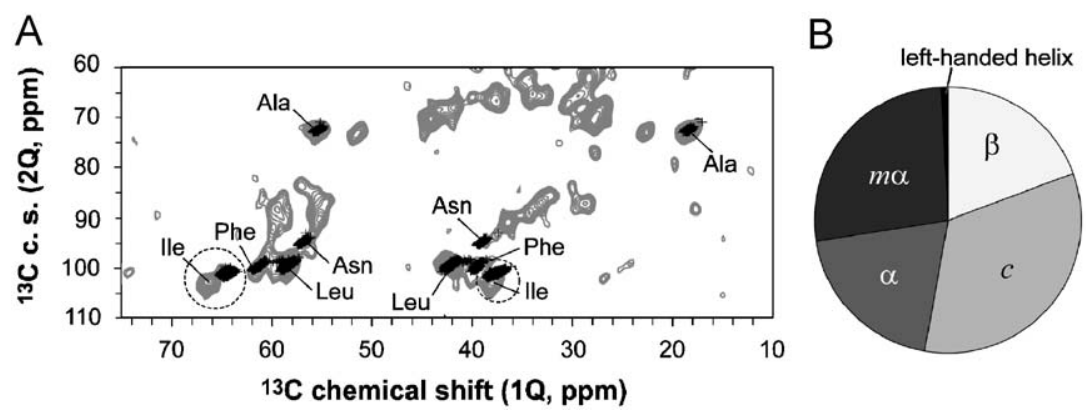

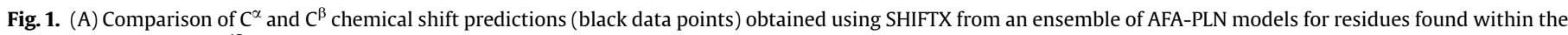

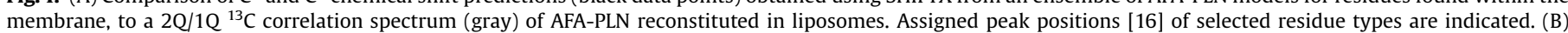


conformations, a sufficiently large number of assignments was available to distinguish between membrane-integral $(m \alpha)$ and not membrane-integral $(\alpha)$ residues.

Table 1

Proteins and polypeptides with solid-state NMR resonance assignments and high-resolution structure information.

\begin{tabular}{|c|c|c|c|c|}
\hline Molecule & Topology & Asgn. res. & ssNMR source, year (BMRB entry) & Prediction source (PDB code, method) \\
\hline BPTI & Globular & 9 & McDermott et al. [25], 2000 & Wlodawer et al. [26] (6PTI, X-ray) \\
\hline Crh & Globular (dimer) & 83 & Bockmann et al. [27], 2003 & Juy et al. [28] (1MU4_A, X-ray) \\
\hline GB1 & Globular & 54 & Franks et al. [29], 2005 & Franks et al. [30] (2GI9, X-ray) \\
\hline KcsA-Kv1.3 & Membrane integral & 72 & Lange et al. [31], 2006; Schneider et al. [32], 2008 & Zhou et al. [29] (1K4C, X-ray), variation [31] \\
\hline KTX & Globular & 26 & Lange et al. [33], 2005 (6341) & Gairi et al. [34] (2KTX_1, soln. NMR) \\
\hline LH2 & Membrane integral & 75 & van Gammeren et al. [24], 2005 (6348) & Papiz et al. [35] (1NKZ_D/E, X-ray) \\
\hline MPX & Membrane bound & 12 & Fujiwara et al. [36], 2004 (6214) & Todokoro et al. [37] (2CZP_1, ssNMR) \\
\hline PLN (AFA) & Membrane integral & 29 & Andronesi et al. [16], 2005 & Andronesi et al. [16] (ssNMR) \\
\hline $\mathrm{SH} 3$ & Globular & 53 & Pauli et al. [38], 2001 & Chevelkov et al. [39] (1U06, X-ray) \\
\hline SRII & Membrane integral & 67 & Etzkorn et al. [40], 2007 & Royant et al. [41] (1H68, X-ray) \\
\hline TRX & Globular & 103 & Marulanda et al. [42], 2005 & Katti et al. [43] (2TRX, X-ray) \\
\hline TTR & Fibril & 9 & Jaroniec et al. [44], 2004 & Jaroniec et al. [44], 2004 (1RVS_1, ssNMR) \\
\hline UBI & Globular & 63 & Seidel et al. [45], 2005 & Cornilescu et al. [46] (1D3Z_1, soln. NMR) \\
\hline
\end{tabular}

Column three shows the number of residues in the final data base with at least one assigned resonance. 
correlations) was used. Chemical shift predictions were rereferenced for each protein and each program individually to yield zero offset for the prediction average, with a common offset for all three carbons sites. For this purpose, predictions that deviated by more than $3 \mathrm{ppm}\left({ }^{13} \mathrm{C}\right)$ or $6 \mathrm{ppm}\left({ }^{15} \mathrm{~N}\right)$ from the experimental values were temporarily disregarded here. In order to handle reference offsets consistently, ssNMR data was first adjusted to yield minimum offset with respect to one program (SHIFTX), and then all other predictions were adjusted with respect to the re-referenced experimental data. The final database comprised $512 \mathrm{~N}, 448 \mathrm{C}^{\prime}, 648 \mathrm{C}^{\alpha}$ and $575 \mathrm{C}^{\beta}$ assignments. Overall, 655 residues had at least one solid-state NMR assignment $\left({ }^{13} \mathrm{C}\right.$ or ${ }^{15} \mathrm{~N}$ ). The relative population of secondary structure elements for residues was $20 \% \beta$-strand ( $\beta$ ), 33\% coil (c) and $47 \% \alpha$-helix, with $20 \%$ contributed by not-membrane-integral $(\alpha)$ and $27 \%$ membrane-integral $(m \alpha)$ residues, see Fig. 1B. Five residues (1\%) with assigned chemical shifts found in a left-handed helix were not considered any further.

One protein, the light-harvesting complex LH2 (subunits alpha and beta), showed an unusually large number of incorrect predictions by all four programs, and secondary chemical shifts calculated from the ssNMR assignments were often inconsistent with $\alpha$-helical structure. We attribute these deviations to the presence of paramagnetic ions, and excluded LH2 chemical shifts from our statistical analysis unless stated otherwise. The reduced database comprised of $(22 \%, 35 \%, 21 \%, 21 \%)$ residues in $(\beta, c, \alpha, m \alpha)$ secondary structure conformation, with 580 residues having at least one experimentally determined chemical shift assigned.
Fractional accessible surface areas were calculated using VADAR [23].

\section{Results and discussion}

\subsection{Overall quality of predictions}

\subsubsection{Average prediction inaccuracies}

In principle, due to the offset correction applied, the average over all differences between measured and predicted chemical shifts should vanish. However, using a common offset for all three carbons sites $\left(\mathrm{C}^{\prime}, \mathrm{C}^{\alpha}\right.$ and $\left.\mathrm{C}^{\beta}\right)$, and temporarily disregarding here residues with differences between experimental and predicted shifts by more than $6 \mathrm{ppm}\left({ }^{15} \mathrm{~N}\right)$ or $3 \mathrm{ppm}\left({ }^{13} \mathrm{C}\right), \mathrm{C}^{\prime}$ shifts were usually over-predicted by about $0.1 \mathrm{ppm}$, while $C^{\beta}$ were underpredicted by about the same value, while the average $C^{\alpha}$ prediction was accurate. This effect was negligible for SHIFTX and SPARTA. It was slightly more pronounced when including outliers. Obviously, the average deviation for ${ }^{15} \mathrm{~N}$ vanishes, due to the offset correction. Including ${ }^{15} \mathrm{~N}$ outliers leads to an average inaccuracy of $0.4 \mathrm{ppm}$ for SHIFTS, while the value remains below $0.1 \mathrm{ppm}$ for the other three programs. This indicates that predicting ${ }^{15} \mathrm{~N}$ chemical shifts is more difficult with SHIFTS than for the other programs, in line with other results discussed below.

\subsubsection{Correlation}

For all four programs, predicted and measured solid-state chemical shift were clearly correlated, as can be seen in Fig. 2.


Relative experimental SSNMR chemical shift $\delta_{\exp }{ }^{(\mathrm{SCS})}(\mathrm{ppm})$




regression and the diagonal of perfect predictions are given as solid lines. Note the different scale for ${ }^{15} \mathrm{~N}$. The RMS prediction error is additionally shown in Fig. 3 . 
Residue-specific offsets were removed by subtracting from each predicted and experimentally determined chemical shift the random coil chemical shift for the respective amino acid residue type. For this purpose, we calculated the differences $\Delta \delta_{\exp }^{(\mathrm{SCS})}=$ $\delta_{\exp }-\delta_{\text {rc }}$ (res) and $\Delta \delta_{\text {pred }}^{\text {(SCS) }}=\delta_{\exp }-\delta_{\text {rc }}$ (res), where $\delta_{\exp }$ denotes the experimentally determined chemical shift, and $\delta_{\text {rc }}$ (res) the random coil chemical shift obtained from Ref. [21]. With this definition, $\Delta \delta_{\exp }^{(\mathrm{SCS})}$ and $\Delta \delta_{\text {pred }}^{(\mathrm{SCS})}$ correspond to what is commonly referred to as 'secondary chemical shift'. Also, the correlation coefficients for different carbons and nitrogen can be compared more easily than in absolute terms, since any bias by variations in chemical shift dispersion, e.g. of $\mathrm{C}^{\beta}$ (range of ca. $60 \mathrm{ppm}$ ) compared to $\mathrm{C}^{\prime}$ (range of ca. $15 \mathrm{ppm}$ ) is removed. All four programs show a slight tendency to under-estimate large shift deviations from the reference value, see for example correlations for $\mathrm{C}^{\alpha}$ near 5-6 ppm in Fig. 2. Chemical shifts for ${ }^{15} \mathrm{~N}$ deviate much more strongly from the random coil value than ${ }^{13} \mathrm{C}$; note the different scales for ${ }^{15} \mathrm{~N}$ and ${ }^{13} \mathrm{C}$ in Fig. 2. The (Pearson) correlation coefficient $R$ for SHIFTX and SPARTA predictions was about 0.82 for ${ }^{15} \mathrm{~N}$, and 0.87 for $\mathrm{C}^{\alpha}$, see Fig. 2 and Table 2 . $R$ is smaller for $\mathrm{C}^{\prime}$ and $C^{\beta}$, i.e., the correlations are less good. The same trend is observed for SHIFTS and PROSHIFT, however, with smaller correlation coefficients $R$.

\subsection{Mean prediction error}

The root-mean-square deviation (RMSD) of predictions from experimental shifts is $3 \mathrm{ppm}$ for ${ }^{15} \mathrm{~N}$ and around $1.4 \mathrm{ppm}$ for ${ }^{13} \mathrm{C}$ for SHIFTX and SPARTA, larger for PROSHIFT, and largest for SHIFTS, see Table 2 and Fig. 3. In terms of RMSD, SHIFTX performs about $30 \%\left({ }^{15} \mathrm{~N}\right)$ and about $15 \%\left({ }^{13} \mathrm{C}\right)$ better than SHIFTS. SPARTA performs essentially as well as SHIFTX for ${ }^{15} \mathrm{~N}$, but about $6 \%$ better for ${ }^{13} \mathrm{C}$. With respect to predictions for solvated proteins, carbon predictions are about $\frac{1}{4}$ less precise, as can be seen in Fig. 3 from the comparison to SPARTA statistics of solution-state NMR test predictions [12].

Notably, deviations of chemical shift predictions from experimentally determined values do not necessarily result from a methodological shortcoming, but may also indicate a difference between the prediction input model, in most cases a solution-state NMR or X-ray diffraction structure, and the molecular structure of the sample in the solid-state NMR experiment. In addition to conformational effects of the molecule of interest itself, the molecular environment may possess a significant influence on chemical shifts. For example, this can be seen for the LH2 complex subunits alpha and beta [24], which apparently experience chemical shift distortions from the paramagnetic co-factor. Inclusion of LH2 in the database leads to an average ${ }^{13} \mathrm{C}$ prediction RMSD increase of almost $10 \%$, as well as a reduction of the correlation coefficient $R$ by $4 \%$. This is in line with the observation of poor correspondence between TALOS [17] backbone torsion angle predictions and the X-ray backbone structure [24].

\subsection{Outliers}

Predictions were further inspected regarding the probability of differing from the experimentally determined shift by more than a certain threshold. For example, shift differences between $\beta$-strands and $\alpha$-helices typically exceed $3 \mathrm{ppm}$ for backbone carbons, with coil values found in between [21]. It is hence important to know how reliably predictions can be found within such a shift range. We observed a fraction of $4 \%$ of ${ }^{13} \mathrm{C}$ predictions differing from experimental values by more than $3 \mathrm{ppm}$ for SPARTA, see Table 2. In line with an increased RMSD, more SHIFTX, PROSHIFT and SHIFTS predictions exceed the threshold. This is in agreement with a statistical normal distribution, with the threshold chosen here being equivalent to about two standard deviations. While these results are quite encouraging, the situation was found much worse for ${ }^{15} \mathrm{~N}$, in which case $82 \%$ of SHIFTS predictions deviated from the experimental values by more than $3 \mathrm{ppm}$, see Table 2 . Even in the best case, namely for

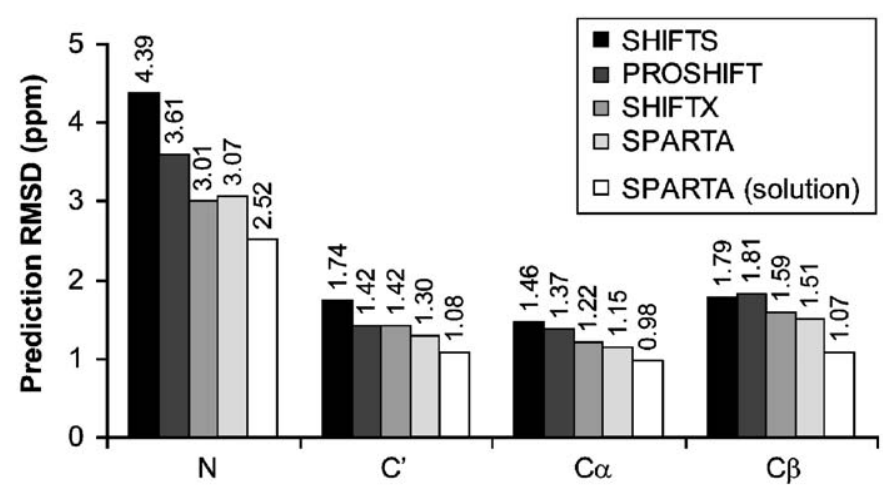

Fig. 3. Comparison of root-mean square prediction errors for SHIFTS, PROSHIFT, SHIFTX and SPARTA with respect to SSNMR data. Values for SPARTA predictions of solution-state NMR chemical shifts [12] are given as a reference.

Table 2

Summary of indicators for the quality of solid-state NMR chemical shift predictions.

\begin{tabular}{|c|c|c|c|c|c|}
\hline Indicator & Program & $N$ & $\mathrm{C}^{\prime}$ & $C^{\alpha}$ & $C^{\beta}$ \\
\hline \multirow[t]{4}{*}{ Outliers (\%) } & SHIFTS & $15(82)$ & 10 & 6 & 9 \\
\hline & PROSHIFT & $11(49)$ & 3 & 5 & 10 \\
\hline & SHIFTX & $6(35)$ & 4 & 4 & 6 \\
\hline & SPARTA & $6(37)$ & 2 & 3 & 5 \\
\hline For threshold (ppm) & & $6(3)$ & 3 & 3 & 3 \\
\hline \multirow[t]{4}{*}{ Prediction RMSD (ppm) (including outliers), see also Fig. 3} & SHIFTS & 4.4 & 1.7 & 1.5 & 1.8 \\
\hline & PROSHIFT & 3.6 & 1.4 & 1.4 & 1.8 \\
\hline & SHIFTX & 3.0 & 1.4 & 1.2 & 1.6 \\
\hline & SPARTA & 3.1 & 1.3 & 1.1 & 1.5 \\
\hline Solution (Ref. [12]) & SPARTA & 2.5 & 1.1 & 1.0 & 1.1 \\
\hline \multirow[t]{4}{*}{ Regression coefficient $R$ (including outliers) } & SHIFTS & 0.69 & 0.58 & 0.80 & 0.63 \\
\hline & PROSHIFT & 0.74 & 0.64 & 0.83 & 0.59 \\
\hline & SHIFTX & 0.83 & 0.68 & 0.86 & 0.70 \\
\hline & SPARTA & 0.82 & 0.72 & 0.88 & 0.74 \\
\hline Solution (Ref. [12]) & SPARTA & 0.79 & 0.83 & 0.91 & 0.82 \\
\hline
\end{tabular}


SHIFTX, just $65 \%$ of predictions are within threshold, owing to the larger RMS prediction error for nitrogen with respect to carbons. If the threshold for ${ }^{15} \mathrm{~N}$ is doubled, the number of outliers falls to $15 \%$ for SHIFTS, and is as low as $6 \%$ for SHIFTX and SPARTA, with PROSHIFT again ranging in between, see Table 2. While showing the fewest outliers, SHIFTX and SPARTA predictions are also most precise (Table 2), again in agreement with a normal distribution of deviations. The increased probability of predicting a chemical shift within a given cutoff is not trade off for lower precision, encouraging the use of the more recent programs.

The presence of a certain fraction of incorrect predictions is not necessarily a critical aspect when a large number of predictions are being evaluated at the same time, allowing for statistical outliers, such as in context of general data assessment or structure fitting to spectra [13-15]. In this case, quantitative information as presented above can be helpful in adjusting the weight of chemical shift contributions to structural fitting algorithms. On the other hand, the correctness of individual predictions limits unambiguous identifications of individual cross peaks in a spectrum. In these cases, reliable chemical shift identifications can only be obtained from sequential assignment by spectroscopic means.

\subsection{Influence of secondary structure and chemical environment}

To first order, nuclei exhibit a particular chemical shift depending on the covalent chemical structure given by each amino acid residue, for example with $\mathrm{Thr} \mathrm{C}^{\beta}$ being vastly different from Ala $C^{\beta}$. Such residue-specific average chemical shifts have been empirically collected and, for example, tabulated in the BioMagResBank [19]. This residue-specific average can be further refined by dividing residues into classes according to their secondary structure. We consider in the following these residue-specific, secondary-structure dependent average values as reported by Wang and Jardetzky [21]. Essentially, these tabulated values can be used to 'manually' predict spectra similar to the four programs considered here, based on secondary structure information alone. To find out how much a computer-based prediction from 3D structure improves on this simplistic approach, we calculated the difference $\Delta \delta_{\exp }^{(\mathrm{WJ})}=\delta_{\exp }-\delta_{\mathrm{WJ}}($ res, ss) and $\Delta \delta_{\text {pred }}^{(\mathrm{WJ})}=\delta_{\text {pred }}-\delta_{\mathrm{WJ}}\left(\right.$ res, ss), where $\delta_{\text {exp }}$ denotes the experimentally determined chemical shift, and $\delta_{\mathrm{wJ}}($ res, ss) the tabulated chemical shift depending on residue type and secondary structure according to Ref. [21]. Again, given a significant fraction of residues in membrane-integral $\alpha$-helices, and motivated by the prediction offset found for some membrane-integral $\mathrm{C}^{\alpha}$ noted above, we treat such residues as a separate class.

Individual distributions of $\mathrm{C}^{\prime}, \mathrm{C}^{\alpha}$ and $\mathrm{C}^{\beta}$ resonances according to the four secondary structure classifications in the database ( $\beta$-strand $(\beta)$, random coil $(c), \alpha$-helix $(\alpha)$ and membrane-integral $\alpha$-helix $(m \alpha)$ ) are shown in Fig. 4 relative to values for $\beta$, c, and $\alpha$ published in Ref. [21] for all four programs. One finds a positive correlation between predicted and measured chemical shift, in other words, there is a benefit from using a chemical shift

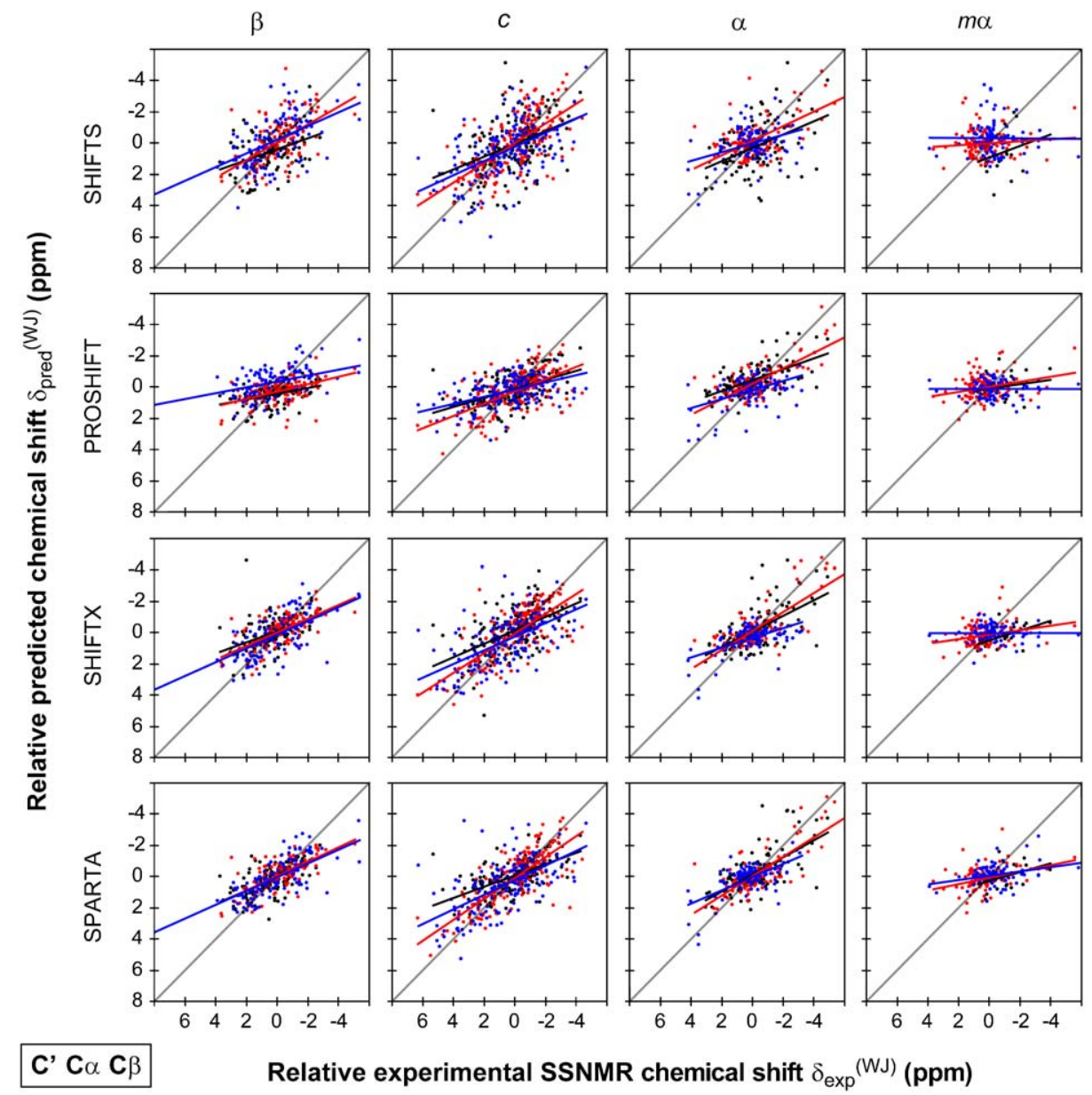

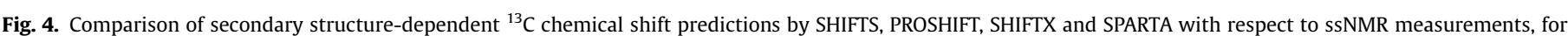

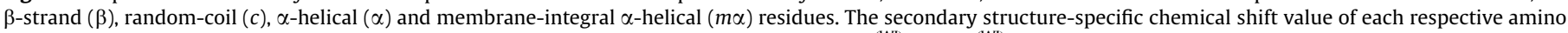


corresponding to perfect predictions are given as solid lines. 
prediction program over the tabulated shifts. Notably, the correlation coefficient of resonance predictions (as indicated by the slope of the regression curve) is generally lower within $\alpha$-helices, in particular membrane-integral $\alpha$-helices, and relatively high for coil structure. This trend can be attributed to rather small shift dispersion within helices, in particular membraneintegral $\alpha$-helices. Furthermore, the correlations are found to be generally less good for $C^{\beta}$ than for $C^{\alpha}$ and $C$. In case of $C^{\beta}$ resonances in membrane-integral $\alpha$-helices for SHIFTS, PROSHIFT and SHIFTX, prediction and measured shift are largely uncorrelated, i.e., none of the programs gives an advantage over tabulated secondary-specific average shifts when predicting from threedimensional structures and the chemical shift prediction reflects only secondary structure.

In addition, there is a trend to under-estimate the magnitude of large chemical shift offsets from the reference value, as can be seen from a larger number of correlation points above the diagonal than below for relative shifts $\Delta \delta_{\exp }^{(\mathrm{WJ})}$ and $\Delta \delta_{\text {pred }}^{(\mathrm{WJ})}$ in the range of 5-6 ppm. This is in line with the observed misalignment of predicted and measured Ile $\mathrm{C}^{\alpha}$ shifts shown in Fig. 1A. In order to reduce such effects, a more detailed identification of deviations is required, for example by considering residue-specific statistics.

\subsection{Dependence on residue surface exposure}

In the solid state, molecules may be subject to molecular interactions different from the solution state. In order to delineate a potential correlation with surface access, which could then be corrected for, we investigated the dependence of prediction RMSD



Fig. 5. Dependence of chemical shift prediction accuracy on residue-specific fractional accessible surface area (ASA) for SHIFTX and SPARTA. Intervals relate to $0-28 \%, 28-52 \%$ and $52-100 \%$ surface exposure and are chosen such that each bin comprises $\frac{1}{3}$ of all residues. Prediction RMSD normalized to values given in Table 2 . on accessible surface area for the two programs with lowest RMSD, SHIFTX and SPARTA. For this purpose, residues were sorted into three equal bins (circa 180 residues per bin) according to their fractional accessible surface area (ASA), calculated with VADAR [23]. Smaller bins did not yield an improvement on the analysis presented here.

According to Fig. 5, there is a variation of prediction RMSD with ASA. SHIFTX prediction accuracies for $\mathrm{N}, \mathrm{C}^{\prime}$ and $\mathrm{C}^{\alpha}$ vary by about $\pm 10 \%$ among the three bins, while $C^{\beta}$ show a stronger variation. In SPARTA, $\mathrm{C}^{\prime}$ varies most, and a qualitative agreement of the trend for $C^{\beta}$ with respect to SHIFTX is observed. Predictions for $C^{\alpha}$ and $\mathrm{C}^{\beta}$ in SHIFTX and all carbons in SPARTA are on average less accurate for residues with high surface accessibility as compared to intermediate ASA values. On the other hand, the trend is not unequivocally continued towards low ASA values. Also, nitrogen chemical shift predictions are least accurate for intermediate ASA values, in contrast to carbons. SPARTA $C^{\prime}$ and $N$ predictions depend on surface accessibility in the opposite way, whereas this is not the case for SHIFTX. In the best cases (near 0.85 normalized RMSD), SPARTA predictions are as good as in solution.

While the dependence of $C^{\alpha}$ prediction RMSD is almost proportional to surface exposure, there appears to be no simple functional relation between RMSD and ASA that could be attributed to a single, surface-related physical parameter, and additionally be corrected for. We see for both programs that nuclei with shifts sensitive to hydrogen bond formation $\left(N, \mathrm{C}^{\prime}\right)$ and sidechain orientation $\left(C^{\beta}\right)$ have the least conclusive behavior in reference to ASA, whereas surface exposure correlates quite well with $C^{\alpha}$ prediction accuracy. This may indicate that other influences mask a potential influence of surface exposure for $N$, $\mathrm{C}^{\prime}$ and $\mathrm{C}^{\beta}$.

\subsection{Residue-specific analysis}

Predictions may be more reliable for some residues than for others. We investigated this by further breaking down the general RMSD analysis to a residue-specific level. Residue-specific differences between predicted and observed chemical shifts are shown in Fig. 6 for $C^{\alpha}$ in SHIFTX. They are, by magnitude, representative also for other resonances $\left(\mathrm{N}, \mathrm{C}^{\prime}, \mathrm{CB}\right)$ and programs (data not shown). Results based on at least $20 \mathrm{C}^{\alpha}$ assignments are shown in black, results based on 10-20 $\mathrm{C}^{\alpha}$ assignments in gray, others white. The average predictions scatter around mean values deviating by $1.2 \mathrm{ppm}$ from the experimentally determined value, as found when all residue types are combined. For each residue type, the scatter is significantly larger than the average deviation. Large inaccuracies, such as for leucine $C^{\prime}$ in SHIFTS, serine $C^{\prime}$ in PROSHIFT, or tryptophan $\mathrm{C}^{\alpha}$ in SPARTA are not found in all



Fig. 6. Residue-specific differences between predicted and observed $\mathrm{C}^{\alpha}$ chemical shifts for SHIFTX, averaging over all secondary structure elements. Black, gray and white bars indicate the availability of at least 20,10 , or less than $10 \mathrm{C}^{\alpha}$ assignments, respectively. 
programs concurrently (data not shown). The same is true for $\mathrm{N}, \mathrm{C}^{\prime}$ and $C^{\beta}$. The prediction accuracy is generally better for residues with a large number of assignments, indicating that these simply reflect insufficient statistical averaging. In principle, however, the correction of residual inaccuracies as presented in Fig. 6 could be used to improve the prediction quality, given a large number of assignments. In practice, this could be achieved by taking ssNMR assignments into account when training the programs.

\subsection{Residue-specific effects from secondary structure and environment}

It would be beneficial for improving the prediction methods to understand if some inaccuracies can be traced back to specific groups of residues of the same kind, such as seen for membraneintegral Ile $\mathrm{C}^{\alpha}$. Therefore, the prediction offset described above motivated us to inspect residue-specific predictions in distinct secondary structure elements $(\alpha, c, \beta, m \alpha)$, where again membrane-integral $\alpha$-helices were considered separately. A further discrimination of residue-specific shift predictions according to the four distinguished classes $(\alpha, c, \beta, m \alpha)$, however, is difficult due to the low number of assignments available for the same residue type and structure class. In many cases, less than 10 assignments were available, making a statistical assessment unreliable. Where at least 20 assignments per residue and structure class were present, deviations from measured values lie within the prediction error, provided the shift offset for ${ }^{15} \mathrm{~N}$ is adjusted for each program individually (data not shown). When at least 10 , but less than 20 assignments are available, even with LH2 included in the database, the only exception is Val $C^{\alpha}(m \alpha)$ with an inaccuracy of $-0.77 \mathrm{ppm}$ at an RMSD of $0.63 \mathrm{ppm}$ for SHIFTX, but only a slightly similar trend observed with SPARTA. This observation is based on 10 assignments only, and should be revised when data for other membrane-integral proteins will be available. Predictions for $\mathrm{C}^{\alpha}$ of $\alpha$-helical Ile residues within membranes exhibit a trend to under-estimate the measured shift, which is most pronounced in SHIFTX, see Fig. 7 (black bars). Average over 22 assigned residues, with LH2 included, the SHIFTX prediction shows an offset of $-0.74 \mathrm{ppm}$ with an RMSD of $1.95 \mathrm{ppm}$. This, however, is not representative of the Ile $C^{\alpha}$ mismatch exemplified in Fig. 1, wherein the scatter is clearly smaller than the mean offset. Interestingly, the prediction RMSD is about three times larger than for Gly and Ala, for example, where a large number of assignments are available, too. A detailed look into the data reveals that there are just three residues that do not follow the general trend: For I26 in LH2 subunit $\alpha$, I16 in LH2 subunit $\beta$ and I33 in AFA-PLN, shift predictions are off by 2.4, 3.3 and $5.2 \mathrm{ppm}$. In contrary, all other membrane-integral Ile $C^{\alpha}$ shifts are off by roughly $-2 \mathrm{ppm}$, including other LH2 and AFA-PLN predictions

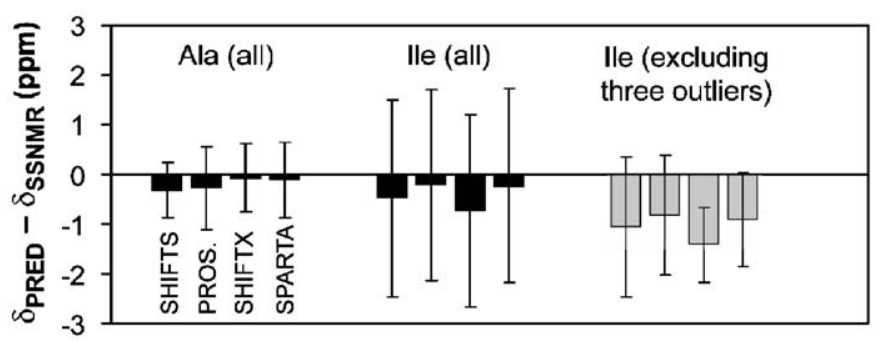

Fig. 7. Inaccuracy of Ile $C^{\alpha}$ predictions in membrane-integral $\alpha$-helices for (left to right) SHIFTS, PROSHIFT, SHIFTX and SPARTA, with Ala $\mathrm{C}^{\alpha}$ as a reference. Here, shifts of LH2 were included in the analysis. Results before and after exclusion of three outliers out of 22 residues, as discussed in the text, are shown in black and gray, respectively.
(SHIFTX). These prediction outliers are also seen for all other programs, and the respective input structures do not show unusual backbone torsion angles at these positions. Hence, the outliers may point to interactions with other molecules, such as cofactors in LH2, or lipid head groups in case of AFA-PLN, provided the assignment is correct. Exclusion of these $C^{\alpha}$ predictions from the database finally leads to an average prediction inaccuracy of $-1.42 \mathrm{ppm}$ with a relatively high precision of $0.75 \mathrm{ppm}$ for the remaining 19 assignments, see Fig. 7 (gray bars). This is representative for the Ile $\mathrm{C}^{\alpha}$ mismatch shown in Fig. 1. The same trend can be observed for SHIFTS, PROSHIFT and SPARTA.

\subsection{Joining forces}

Last, one might ask the question if the performance of shift predictions can be improved by combined analysis using all four programs together. Indeed, averaging over SHIFTX and SPARTA predictions very slightly improves the results. The number of outliers beyond a threshold of more than $6 \mathrm{ppm}$ for $\mathrm{N}$ and $3 \mathrm{ppm}$ for ${ }^{13} \mathrm{C}$, as described for the individual programs in Table 2, is similar to SPARTA with $(5 \%, 3 \%, 3 \%, 5 \%)$. The RMSD is slightly decreased for ${ }^{15} \mathrm{~N}$, and constant for ${ }^{13} \mathrm{C}$ with $(2.9,1.3,1.1,1.5) \mathrm{ppm}$, and $R$ has marginally improved to $(0.84,0.73,0.88,0.74)$. In the end, the gain is negligible for practical use, but indicates that further improvement in prediction methodology should be possible.

\section{Conclusions}

Protein NMR chemical shift prediction computer programs trained with solution-state NMR chemical shifts and solutionstate NMR or X-ray structural data are a valid tool for predicting the chemical shift measured by ssNMR spectroscopy. For the best programs, the RMS prediction error is found to be in the order of $3 \mathrm{ppm}$ for ${ }^{15} \mathrm{~N}$ and $1.5 \mathrm{ppm}$ for ${ }^{13} \mathrm{C}$, with a chance of about $93 \%$ and $95 \%$ of finding the predicted shift within an interval of 6 and $3 \mathrm{ppm}$ around the experimentally determined value for ${ }^{15} \mathrm{~N}$ and ${ }^{13} \mathrm{C}$, respectively. Including outliers, predictions are about $\frac{1}{4}$ less precise than for proteins in solution. The decreased precision may partially be attributed to surface effects. Generally, highest precision is achieved for $\mathrm{C}^{\alpha}$. Correlation coefficients are influenced by resonance, secondary structure type, and also by the molecular environment, with a tendency to under-estimate large chemical shift offsets from the residue-specific average. Given a sufficiently large number of assignments, none of the considered computer programs shows prediction inaccuracies that exceed the prediction RMSD. However, taking residual inaccuracies into account, predictions could slightly be improved. Unusual deviations from experimental values did occur in certain combinations of residue type and secondary structure or molecular environment, and should be reconsidered when more protein assignments become available. In case of Ile $\mathrm{C}^{\alpha}$ in membrane-integral, $\alpha$-helical residues, the deviations of SHIFTX predictions from measured chemical shifts become significant when three unusual outliers are excluded from the database, and a clear trend of underpredicting this shift is then also visible in SHIFTS, PROSHIFT and SPARTA. These predictions may be corrected manually. A possible methodological improvement is indicated by a slightly better performance of SHIFTX and SPARTA combined. The use of SPARTA or SHIFTX is encouraged, where SPARTA is found to be slightly more precise and reliable. On the other hand, when speed is a critical objective, such as for applications within a structure calculation framework, SHIFTX provides optimum performance per time. 


\section{Acknowledgment}

We are grateful to thank Gitta Angerstein for technical assistance.

\section{References}

[1] I. Ando, H. Saito, R. Tabeta, A. Shoji, T. Ozaki, Macromolecules 17 (1984) 457-461.

[2] A.C. Dedios, J.G. Pearson, E. Oldfield, Science 260 (1993) 1491-1496.

[3] H. Saito, Magn. Reson. Chem. 24 (1986) 835-852.

[4] S. Spera, A. Bax, J. Am. Chem. Soc. 113 (1991) 5490-5492.

[5] D.S. Wishart, B.D. Sykes, F.M. Richards, J. Mol. Biol. 222 (1991) 311-333.

[6] H.B. Le, E. Oldfield, J. Biomol. NMR 4 (1994) 341-348.

[7] J. Glushka, M. Lee, S. Coffin, D. Cowburn, J. Am. Chem. Soc. 111 (1989) 7716-7722.

[8] M. Iwadate, T. Asakura, M.P. Williamson, J. Biomol. NMR 13 (1999) 199-211.

[9] X.P. Xu, D.A. Case, Biopolymers 65 (2002) 408-423.

[10] J. Meiler, J. Biomol. NMR 26 (2003) 25-37.

[11] S. Neal, A.M. Nip, H.Y. Zhang, D.S. Wishart, J. Biomol. NMR 26 (2003) 215-240.

[12] Y. Shen, A. Bax, J. Biomol. NMR 38 (2007) 289-302.

[13] H. Heise, S. Luca, B.L. de Groot, H. Grubmuller, M. Baldus, Biophys. J. 89 (2005) $2113-2120$

[14] Y. Matsuki, H. Akutsu, T. Fujiwara, J. Biomol. NMR 38 (2007) 325-339.

[15] A. Cavalli, X. Salvatella, C.M. Dobson, M. Vendruscolo, Proc. Natl. Acad. Sci. USA 104 (2007) 9615-9620.

[16] O.C. Andronesi, S. Becker, K. Seidel, H. Heise, H.S. Young, M. Baldus, J. Am. Chem. Soc. 127 (2005) 12965-12974.

[17] G. Cornilescu, F. Delaglio, A. Bax, J. Biomol. NMR 13 (1999) 289-302.

[18] M.V. Berjanskii, S. Neal, D.S. Wishart, Nucl. Acids Res. 34 (2006) W63-W69.

[19] B.R. Seavey, E.A. Farr, W.M. Westler, J.L. Markley, J. Biomol. NMR 1 (1991) 217-236.

[20] H.M. Berman, J. Westbrook, Z. Feng, G. Gilliland, T.N. Bhat, H. Weissig, I.N. Shindyalov, P.E. Bourne, Nucl. Acids Res. 28 (2000) 235-242.

[21] Y.J. Wang, O. Jardetzky, Protein Science 11 (2002) 852-861.

[22] Predictions that were found being completely off for SHIFTS showed no obvious correlation with residue type or residue number (such as SH3 S19 or Ubi I36), and input files for six out of seven affected proteins were approved structures from the Protein Data Bank. However, the "self chi effect" contribution was almost 180 instead of a value near zero, suggesting a complication with the angle modulus. While this manuscript was being revised, this error seems to have been corrected.

[23] L. Willard, A. Ranjan, H. Zhang, H. Monzavi, R.F. Boyko, B.D. Sykes, D.S. Wishart, Nucl. Acids Res. 31 (2003) 3316-3319.
[24] A.J. van Gammeren, F.B. Hulsbergen, J.G. Hollander, H.J.M. de Groot, J. Biomol. NMR 31 (2005) 279-293.

[25] A. McDermott, T. Polenova, A. Bockmann, K.W. Zilm, E.K. Paulsen, R.W. Martin, G.T. Montelione, J. Biomol. NMR 16 (2000) 209-219.

[26] A. Wlodawer, J. Nachman, G.L. Gilliland, W. Gallagher, C. Woodward, J. Mol Biol. 198 (1987) 469-480.

[27] A. Bockmann, A. Lange, A. Galinier, S. Luca, N. Giraud, M. Juy, H. Heise, R. Montserret, F. Penin, M. Baldus, J. Biomol. NMR 27 (2003) 323-339.

[28] M. Juy, F. Penin, A. Favier, A. Galinier, R. Montserret, R. Haser, J. Deutscher, A. Bockmann, J. Mol. Biol. 332 (2003) 767-776.

[29] W.T. Franks, D.H. Zhou, B.J. Wylie, B.G. Money, D.T. Graesser, H.L. Frericks G. Sahota, C.M. Rienstra, J. Am. Chem. Soc. 127 (2005) 12291-12305.

[30] W.T. Franks, B.J. Wylie, S.A. Stellfox, C.M. Rienstra, J. Am. Chem. Soc. 128 (2006) 3154-3155.

[31] A. Lange, K. Giller, S. Hornig, M.F. Martin-Eauclaire, O. Pongs, S. Becker M. Baldus, Nature 440 (2006) 959-962

[32] R. Schneider, C. Ader, A. Lange, K. Giller, S. Hornig, O. Pongs, S. Becker, M. Baldus, J. Am. Chem Soc. 130 (2008) 7427-7435.

[33] A. Lange, S. Becker, K. Seidel, K. Giller, O. Pongs, M. Baldus, Angew. Chem. Int Ed. Engl. 44 (2005) 2089-2092.

[34] M. Gairi, R. Romi, I. Fernandez, H. Rochat, M.F. Martin-Eauclaire, J. Van Rietschoten, M. Pons, E. Giralt, J. Pept. Sci. 3 (1997) 314-319.

[35] M.Z. Papiz, S.M. Prince, T. Howard, R.J. Cogdell, N.W. Isaacs, J. Mol. Biol. 326 (2003) 1523-1538.

[36] T. Fujiwara, Y. Todokoro, H. Yanagishita, M. Tawarayama, T. Kohno K. Wakamatsu, H. Akutsu, J. Biomol. NMR 28 (2004) 311-325.

[37] Y. Todokoro, I. Yumen, K. Fukushima, S.W. Kang, J.S. Park, T. Kohno, K. Wakamatsu, H. Akutsu, T. Fujiwara, Biophys. J. 91 (2006) 1368-1379.

[38] J. Pauli, M. Baldus, B. van Rossum, H. de Groot, H. Oschkinat, ChemBiochem 2 (2001) 272-281.

[39] V. Chevelkov, K. Faelber, A. Diehl, U. Heinemann, H. Oschkinat, B. Reif J. Biomol. NMR 31 (2005) 295-310.

[40] M. Etzkorn, S. Martell, O.C. Andronesi, K. Seidel, M. Engelhard, M. Baldus, Angew. Chem. Int. Ed. Engl. 46 (2007) 459-462.

[41] A. Royant, P. Nollert, K. Edman, R. Neutze, E.M. Landau, E. Pebay-Peyroula, J. Navarro, Proc. Natl. Acad. Sci. USA 98 (2001) 10131-10136.

[42] D. Marulanda, M.L. Tasayco, M. Cataldi, V. Arriaran, T. Polenova, J. Phys. Chem. B 109 (2005) 18135-18145.

[43] S.K. Katti, D.M. Lemaster, H. Eklund, J. Mol. Biol. 212 (1990) 167-184.

[44] C.P. Jaroniec, C.E. MacPhee, V.S. Bajaj, M.T. McMahon, C.M. Dobson, R.G. Griffin, Proc. Natl. Acad. Sci. USA 101 (2004) 711-716.

[45] K. Seidel, M. Etzkorn, H. Heise, S. Becker, M. Baldus, ChemBiochem 6 (2005) 1638-1647.

[46] G. Cornilescu, J.L. Marquardt, M. Ottiger, A. Bax, J. Am. Chem. Soc. 120 (1998) 6836-6837. 